\title{
Substance Use Disorder And Quality Of Life Of Clients At An Addiction Rehabilitation Center In Nigeria
}

\author{
${ }^{1}$ Armiya'u .Y. Aishatu(MBBS, FMCPsych, FWACP) ${ }^{2}$ Abdulmalik Jibril(MBBS, \\ FWACP) ${ }^{2}$ Makanjuola .A. Victor (MBBS, FWACP) ${ }^{3}$ Ogunlesi \\ Adegboyega(MBBS, FMCPsych, FWACP, FRCPsych) \\ ${ }^{1}$ Department Of Psychiatry, Jos University Teaching Hospital, Plateau State Nigeria. \\ ${ }^{2}$ Department Of Psychiatry, University College Hospital Ibadan, Oyo State Nigeria. \\ ${ }^{3}$ Federal Neuropsychiatry Hospital Aro Abeokuta, OgunState Nigeria.
}

\begin{abstract}
Background: Substance use disorders are increasingly viewed as a chronic condition, and addiction treatment services are beginning to adopt models that were developed to address other chronic conditions. From this perspective, treatment for addiction aims for the broad goal of recovery, which is defined as abstinence plus improved quality of life. The aim of this study was to assess the subjective and objective quality of life and assess for any differencebetween clients abusing/dependent on single versus multiple drug(s) and/or alcohol at a rehabilitation center in Nigeria.

Method: A cross sectional descriptive design was used to assess 190 clients who consented to be studied. Each of the recruited client was interviewed and completed a socio-demographic, subjective quality of life proforma questionnaire and the WHOQOL-BREF questionnaire. All the respondents also completed an interview session with the Composite International Diagnostic Interview Schedule (CIDI). The data was analyzed using the Statistical Package for Social Sciences Version 20.

Result: Seventy respondents (36.8\%) used multiple substances, while $120(63.2 \%)$ used single substance. Alcohol was the single most abused substance by 111 (40.7\%) respondents; followed by marijuana, 98 (35.9\%). More than half (64.7\%) of the respondents lived in medium density neighbourhood, and a small percentage experienced depressed mood frequently. On the WHOQOL-BREF quality of life scale, respondents scored poorly on the overall general health, domain 1 (physical health) and domain 4 (environment) while on domain 3 (social) subjects had a good score.
\end{abstract}

Conclusion: Clients with substance use disorders tended to have a poorer quality of life.

Keywords: Quality of life, Rehabilitation center, Nigeria, Substance Use Disorder, Prevalence

\section{Introduction}

Drug and alcohol problems are chronic relapsing disorders. There are many harmful and disabling effects from these disorders, not only to the users but also to their families and to the society in general. For some patients, entry into treatment occurs at a time of withdrawal from drugs following the development of dependence. Individuals admitted to a rehabilitation service have often been using drugs and alcohol for many years and are frequently ill [1].

Active substance abuse affects nearly all areas of functioning: vocational, social/familial, physical and mental health, residential status, and access to services [2]. For these and other reasons, substance abusers seek help to quit drugs not only as an end in itself, but as means to escape these consequences and to gain a better life [2]. Therefore, while substance abuse treatment seeks to promote abstinence or at least significant reduction in substance use, its ultimate aim is to improve the patients' quality of life [2]. Quality of life is increasingly recognized as central to the broad construct of recovery in substance abuse services and its measures can supplement more objective symptom measures, identify specific service needs and document changes in functioning that are associated with substance use patterns [3].

Substance use disorder is a chronic condition for most affected individuals and quality of life improvement is a particularly important goal in treating conditions that cannot be cured [2]. Quality of life measurement in a chronic illness framework intends to capture the full impact of a medical condition and recommended treatment for an individual [4]. As a commonly used outcome measure in chronic illnesses, quality of life provides an empirical assessment of patients' experience, functioning and the burden of disease after treatment which is useful information for public health evaluation and for service development and evaluation [5].

Drug and alcohol problems are thought to cause considerable disability and changes to the quality of life of an individual [1]. These are important indices that may help change the perception, treatment and care of those with 
drug and alcohol problems. Muldoon et al stated that there are over 1000 new articles indexed annually under the heading "Quality of life" (QOL) [6].

\section{Study Design and Location}

\section{Methods}

This was a cross-sectional, descriptive study conducted in two stages among 190 clients at the Substance Abuse Treatment and Rehabilitation Center, Plateau state.

\section{Data Collection}

In the first stage, every client was given a questionnaire booklet consisting of a socio-demographic, subjective quality of life, and WHO-QOL-BREF questionnaire, an instrument that has been validated among various populations and used in this environment [7]. The second stage consisted of the substance use disorder module of the CIDI. The instrument has been validated, and used by researchers in different countries including Nigeria [8-11].

\section{Socio-demographic questionnaire}

\section{Instruments}

The socio-demographic questionnaire was designed by the authors to elicit information from the subjects on variables such as age, gender, marital status, and highest level of education among others. The subjective quality of life questionnaire was used to elicit information about the respondents' neighbourhood, access to social amenities among others. The questionnaire is unstructured and was used by Adeponle in a study conducted in Nigeria [12].

\section{World Health Organization Quality of Life - BREF (WHOQOL-BREF)}

This instrument was used to collect data on the objective quality of life of all consenting respondents. It is a short version of the self-report questionnaire (WHOQOL - 100) designed by the WHO to measure individual's perception of their position in life in the context of the culture and value systems in which they live and in relation to their goals, expectations, standards and concerns [13].

\section{Composite International Diagnostic Interview (CIDI)}

The Substance Section was administered to all the clients who consented to participate in the study and fulfill the inclusion criteria. CIDI is a comprehensive, fully standardized interview that can be used to assess mental disorder and provide diagnosis according to the definition and criteria of the tenth revision of the International Classification of Diseases (ICD-10) and the fourth edition of the Diagnostic and Statistical Manual of mental disorder (DSM IV) American Psychiatric Association [10, 14].

\section{Analysis}

Data was analyzed using the Statistical Package for Social Sciences (SPSS) version 20 [15]. The frequency distribution of these variables, means and standard deviation were calculated, and a probability of $5 \%$ was regarded as statistically significant.

\section{Socio-demographic characteristics of respondents}

\section{Results}

Respondents were aged between 13 and 55years with a mean age of 27.5 \pm 8.3 years. More than half of the respondents were between the ages of 20-29 years. Majority of the respondents $155(82 \%)$ were never married, with one hundred and thirteen $(59 \%)$ having some form of tertiary education. A quarter of the respondents were students at the time of the study (Table 1).

Table 1

\begin{tabular}{|l|l|l|}
\hline Demographic variables & $\mathrm{N}=190$ Frequency & Percentage \\
\hline Age group (years) & & \\
$10-19$ & 35 & 18.4 \\
$20-29$ & 98 & 51.6 \\
$30-39$ & 45 & 23.7 \\
$40-49$ & 8 & 4.2 \\
$50-59$ & 4 & 2.1 \\
\hline Marital status & & \\
Married & 23 & 12.1 \\
Separated & 12 & 6.3 \\
\hline
\end{tabular}


Substance Use Disorder And Quality Of Life Of Clients At An Addiction Rehabilitation...

\begin{tabular}{|l|l|l|}
\hline Never married & 155 & 81.6 \\
\hline Educational status & & \\
Non formal & 5 & 2.6 \\
Primary & 15 & 7.9 \\
Secondary & 57 & 30.0 \\
Tertiary & 113 & 59.5 \\
\hline Employment status & \\
Unemployed & & 22.6 \\
Employed & 43 & 31.1 \\
Student & 59 & 40.5 \\
Others & 77 & 5.8 \\
\hline
\end{tabular}

\section{Prevalence of psychoactive substance use in respondents}

Alcohol was the single most abused substance by 78 (41.0\%) respondents; followed by marijuana, 69 $(36.2 \%)$ (Table 2).

Table 2

\begin{tabular}{|l|l|l|}
\hline \multicolumn{2}{|c|}{ N $=190$} & Percentage \\
\hline Psychoactive substances & Frequency & 36.2 \\
\hline Alcohol Dependence & 69 & 4.8 \\
\hline Alcohol Abuse & 9 & 28.4 \\
\hline Marijuana Dependence & 54 & 7.8 \\
\hline Marijuana Abuse & 15 & 9.3 \\
\hline Tobacco Dependence & 18 & 1.1 \\
\hline Tobacco Abuse & 2 & 3.3 \\
\hline Opiate Dependence & 6 & 1.1 \\
\hline Opiate Abuse & 2 & 1.1 \\
\hline Analgesic Dependence & 2 & 1.5 \\
\hline Analgesic Abuse & 3 & 1.4 \\
\hline Cocaine Dependence & 1 & 1.4 \\
\hline Cocaine Abuse & 1 & 1.4 \\
\hline Solvent Dependence & 1 & 1.4 \\
\hline Solvent Abuse & 1 & 1.4 \\
\hline Stimulant Dependence & 1 & 1.4 \\
\hline Stimulant Abuse & 1 & 1.4 \\
\hline Sedative/Hypnotic Dependence & 1 & 2.9 \\
\hline Sedative/Hypnotic Abuse & 6 & \\
\hline
\end{tabular}

Substance of use

Majority of the respondents abused single substance, 120 (63.2\%) (Figure 1)

Figure 1: Pie chart showing distribution of substance use by respondents

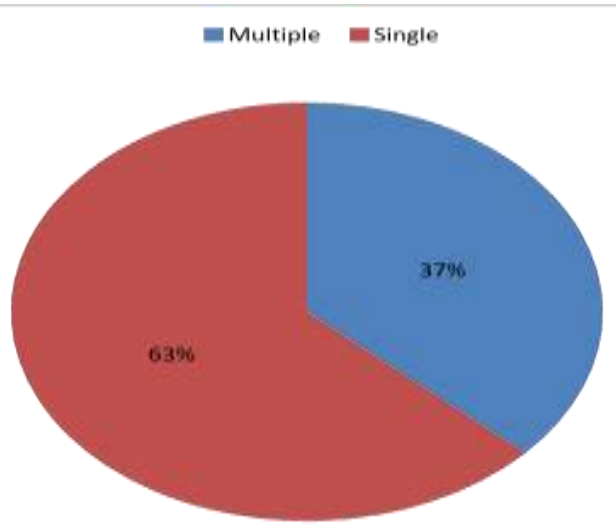

\section{Subjective Quality of Life of respondents}

More than half of the respondents $123(64.7 \%)$ lived in medium density neighborhood with $19(10 \%)$ living in low density neighborhood. Concerning their psychological states, 132(69.5\%) of the respondents sometimes experienced depressed mood, 37(19.5\%) not at all, while 21(11.0\%) experienced depressed mood frequently. One hundred and forty two (74.7\%) experienced anxiety sometimes, 35(18.4\%) did not, while $13(6.9 \%)$ had anxiety symptoms frequently (Figure 2 ) 
Figure 2: Bar chart showing some subjective quality of life of respondents

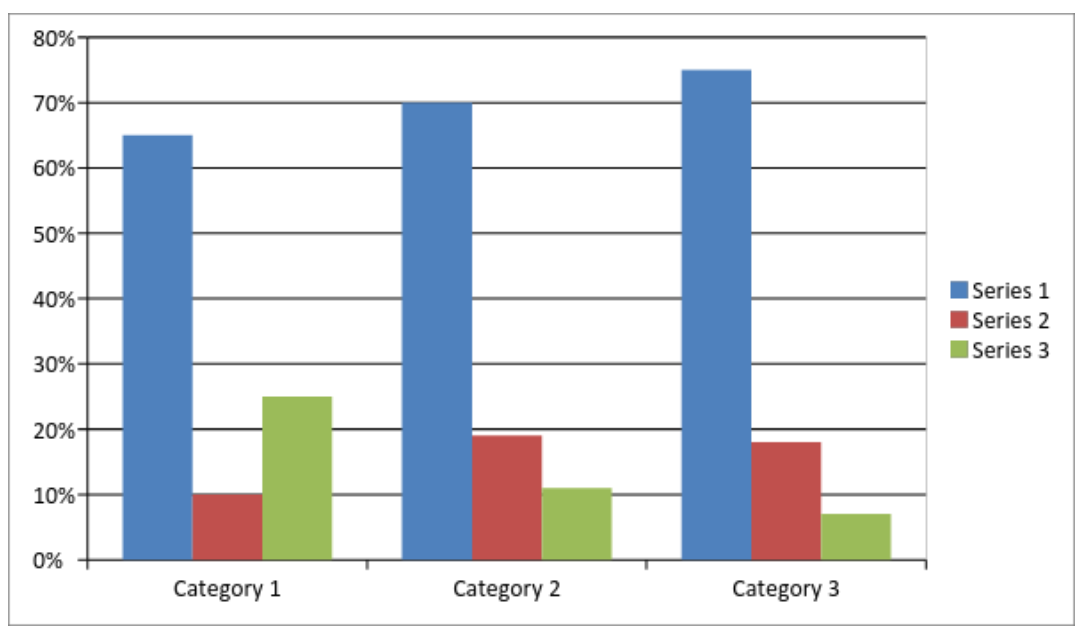

Key:

Category 1:Neighbourhood characteristics (medium, low and high density respectively)

Category 2: Feeling depressed (sometimes, not at all and frequent feelings respectively)

Category 3: Anxious feelings (sometimes, not at all and frequently anxious respectively)

There was no statistically significant relationship was found in any area of the subjective quality of life between respondents abusing single versus multiple substances in this study.

Also no significant findings and no significant relationships were found on the WHOQOL-BREF of the respondents Table 3

Table 3

\begin{tabular}{|l|l|l|l|l|l|}
\hline Domain & $\begin{array}{l}\text { Multiple } \\
\text { Substance } \\
(\mathrm{n}=70)\end{array}$ & $\begin{array}{l}\text { Single Substance } \\
(\mathrm{n}=120)\end{array}$ & $\mathrm{t}$-test & $\mathrm{df}$ & $\mathrm{P}$ \\
\hline Overall quality of life (Q1) & $3.77 \pm 1.02$ & $3.64 \pm 0.95$ & 0.882 & 188 & 0.379 \\
\hline Overall satisfaction with health (Q2) & $3.73 \pm 1.08$ & $3.63 \pm 1.06$ & 0.646 & 188 & 0.519 \\
\hline Domain 1(Physical health) & $13.75 \pm 2.36$ & $13.40 \pm 2.20$ & 1.035 & 188 & 0.302 \\
\hline Domain 2 (Psychological) & $14.10 \pm 2.65$ & $13.88 \pm 2.47$ & 0.556 & 188 & 0.579 \\
\hline Domain 3 (Social relationship) & $14.29 \pm 3.52$ & $14.06 \pm 3.25$ & 0.457 & 188 & 0.648 \\
\hline Domain 4 (Environmental) & $13.31 \pm 2.98$ & $13.23 \pm 2.59$ & 0.199 & 188 & 0.842 \\
\hline
\end{tabular}

\section{Discussion}

The majority of the respondents in this study were males, and this gender imbalance with respect to substance abuse is a frequently reported observation [16]. While several explanations have been proffered for this finding, Maccob and Jaklin posited that the difference is due to the innate tendencies of each gender. Males are more likely to display aggression, violence, independence and a sense of adventure - all of which culminate in experimentation and an increased risk of substance abuse [16].

Many studies have consistently shown that substance abuse is a problem of the youth [17-19]. Schukit observed that for most substances, the age period of highest prevalence of abuse as well as the highest quantity of intake for each of the relevant substances occur between mid-teens and mid-twenties [20]. The preponderance of subjects in this study was between $10-29$ years, accounting for more than half of the sample (70\%). Factors which influenced their indulgence in substance abuse are likely to be peer pressure, experimentation, recreation, and adventurous nature of adolescence [20].

The study looked at the educational levels of the subjects. From the distribution it is evident that 3 in 10 of the participants stopped school at the secondary school level, while more than half were in tertiary institutions or graduates. In similar studies, it was found that $(46 \%)$ and $(33.2 \%)$ of the studied sample dropped out from secondary school which coincided with their commencement of substance abuse [18-19]. School performance seems to be inversely linked with adolescent substance abuse [20]. Drug use is eroding school performance among large segments of a given population and it is a major unresolved problem [19].

Employment status of studied samples had been shown to vary with studies from different regions. In this study, almost half of the subjects were students, with about a third employed, and a quarter were unemployed which is similar to a study in Nigeria by Audu et al where most of their sample were students and 
the unemployed, so also a study in Mumbai which reported a quarter of their studied sample as unemployed [2122].

Majority of the subjects abused single substance with alcohol being the single most abused substance. Results of this study indicated that the commonest substances used by the subjects were alcohol (41.0\%), marijuana $(36.2 \%)$ and tobacco $(10.4 \%)$ in order of frequency. Drugs such as cocaine $(2.4 \%)$, opiate $(4.4 \%)$, and solvents $(2.8 \%)$ etc. were used less frequently. A perusal of the results of Hospital based studies in comparison to this study [11, 23-25], suggested that the common substances often abused by the youths are alcohol, cannabis and tobacco. The level of use of alcohol is probably due to the social permissiveness in alcohol use. It is a drug with important social roles and therefore acceptable [26]. Consequently there are few laws governing its usage and restriction within population. Besides, brewing companies go at length to produce and advertise specific alcoholic beverages aimed at wooing the youths into alcohol use [26].

More than half of the subjects lived in medium density neighbourhoods with few living in a low density neighbourhood. Middle and low density neighbourhoods attachment means that community residents have little connection to others in the neighbourhood, parents don't talk to each other, and few community norms exist regarding such issues as curfew and drinking [27]. This community disorganization can occur in middle and upper-middle class neighbourhoods as well as lower socioeconomic settings [27].

The research found that a small percentage (11\%) of the subjects experienced depressed mood frequently which is similar to a study which found that depressive symptoms were present in (18\%) of their subjects [28]. Multiple substance abuse has been noted as one of the risk factors for psychiatric morbidity in substance abuse [29]. This may be due to the pharmacological toxicities of the substances, acute intoxication, or as a consequence of withdrawal from chronic use [29]. Another study examined the co-morbidity of dependence on single and multiple substances with emotional disorders and found that the risk for psychiatric morbidity was greater among individuals who were uniquely abusing cigarette, alcohol or cannabis [28].

A greater number of subjects using single substance reside in high density neighbourhood compared to those abusing multiple substances but not statistically significant. The majority of these subjects use alcohol and neighbourhoods may influence youth alcohol use through indirect pathways. Youths who live in disadvantaged neighbourhoods may have more access to alcohol which has been found to increase alcohol use [30]. Communities marked by low quality housing and high level of disorganization - vandalism, broken windows, and unoccupied dwelling experience higher levels of drug abuse [27]. Factors that contribute to a person's propensity to abuse drug include genes, mental disorder or personality; however, environment plays an important role also in contributing to potential drug abuse [31]. Peer pressure and drug availability are primary environmental factors contributing to drug abuse and addiction, especially for adolescents [31]. Adolescents exposed to a peer group involved with alcohol and other drugs are more likely to take drugs and drinks. Linked to peer environment, is also the issue of accessibility. The easier it is for a person to obtain drugs, the more likely he will use the drugs. High density neighbourhoods with higher levels of drug availability see a higher level of drug abuse among youngsters [31].

Quality of life is an important index that may help change the perception, treatment and care of those with alcohol and drug dependence problem [22]. The present research work showed lower overall quality of life (Q1) and lower overall satisfaction with health (Q2) in subjects using single substance. It also showed lower quality of life in all the 4 domains (physical, psychological, social relationship and environment) for single substance users compared to those abusing multiple substances, but no statistically significant relationship was found in all the items on the 4 domains and, items 1 and 2 questions on the WHOQOL-BREF with substance use status. The means were though slightly lower for the single substance users in all 4 domains, especially domain 2 (psychological health), so also for the overall quality of life and overall satisfaction with health item domains but not statistically significant.

Various studies have shown a similar result especially studies consisting of single substance users. Alcohol related quality of life studies consistently reported lower levels of quality of life in this group of people [32-34]. Alcohol-dependent individuals experienced improvements in quality of life following treatment, specifically in the domains of mental health, social functioning, general health, and physical functioning [35]. Very few studies done on patients with multiple substance abuse reported lower quality of life than single substance abuser [36]. There is sound evidence that both higher levels of drug consumption and the severity of drug dependence correlate with lower quality of life irrespective of the instrument used [37].

\section{Strengths and limitations}

As far as the authors are aware, the study is the first of its type to be conducted in a population of clients in a rehabilitation center focusing on their quality of life in Nigeria. The population in the rehabilitation center was quite large which strengthens the study further more.

Limitations of the study included being a single site study, its cross-sectional design and the lack of objective measure of comorbidity. 


\section{Conclusion}

The study found that the majority of respondents' abused single substance with alcohol dependence being the commonest diagnosis. Gender difference occurred over a wide range of factors with a clear dominance of substance use noted among the male sample, who were mostly students with secondary school education. No statistically significant difference was observed in the overall quality of life (Q1), overall satisfaction with health (Q2) and the remaining four domains of WHOQOL-BREF scale between single and multiple substances abusers.

\section{Acknowledgements}

Our thanks go to all the subjects who participated in the study for their cooperation, as well to all the staff of the Substance Abuse Treatment and Rehabilitation Center, Plateau state.

\section{Compliance with ethical standards}

Funding: No funding was obtained for the study

Conflict of interest: The authors declare that they have no conflict of interest

Ethical approval: Ethical approval was obtained from the Jos University Teaching Hospital and the Head of the Substance Abuse Treatment and Rehabilitation Center. Informed consent was obtained from each of the subjects.

\section{References}

[1]. Philip, G., Greg, W., \&Khelifa, N.A. (2007). Quality of life and disability in alcohol and drug dependent patients undergoing treatment at Depaul House. IeJSME, 1(1), 35- 40

[2]. Laudet, A.B. (2011). The case for consideration in quality of life in addiction research and clinical practice. Addict Sci and Clinic Pract, 6(1), 44-55

[3]. Notes on Quality of Life. (2012). Quality of life Research Unit, University of Toronto. www.gdrc.org/uem/qol-define

[4]. WHOOQL Group. (1995). The World Health Organization Quality of Life assessment (WHOQOL): Position paper from the WHO. Soc Sci Med, 41(10), 1403-1409.

[5]. Mendlowicz, M.V., \& Stein, M.B. (2000). Quality of life in individuals with anxiety disorders. American Journal of Psychiatry, 157(5), $669-682$

[6]. Muldoon, M., Barge, S., Flory, J., \&Manuck, S. (1998). What are quality of life measurements measuring? British Medical Journal, 316, 542-545.

[7]. World Health Organization. (2004). WHOQOL-BREF quality of life assessment: psychometric properties and results of the international field trial. A report from WHOQOL Group. Quality of Life Research, 13, 299-310.

[8]. Agboola, A.A. (2006). A study of psychiatric and co-morbid physical illness among Inmates in Calabar prison. Unpublished fellowship thesis submitted to the West Africa College of Physicians.

[9]. Sakeeb, L.I. (2007). The prevalence of generalized anxiety disorder and major depression among female convicts in a Nigerian prison. Unpublished fellowship thesis submitted to the West Africa College of Physicians.

[10]. Andrew, G., \& Peter, L. (1998). The psychometric properties of CIDI. Soc PsychiatrEpidemiol, 33(2), 80-88.

[11]. Adamson, T.A., \&Akindele, M.O. (1994). Experience with an in-patient model for alcohol and drug dependence in Nigeria. West African Journal of Medicine, 13(2), $105-8$.

[12]. WHOQOL Group. (1998). WHOQOL Group development of the World Health Organization WHOQOL-BREF quality of life assessment. Psychol Med, 28, 551-558

[13]. Da Silva Lima, A., Fleck, M., Pechansky, F., de Boni, \&Sukop, P.P. (2005). Psychometric Properties of the World Health Organization, quality of life instrument in alcoholic males - a pilot study. Quality of Life Research, 14, 473-478

[14]. American Psychiatric Association. (1994). Fourth Edition of the Diagnostic and Statistical Manual of Mental Disorder (DSM IV) 1994

[15]. Statistical Package for Social Sciences (SPSS 17.0). Chicago IL, USA.

[16]. Maccoby, E.E., \&Jacklin, C.N. (1974). The psychology of sex difference. Standford, USA: Standford University Press.

[17]. Webb, E., Ashton, C.H. \&Kamali, F. (1996). Alcohol and drug use in U.K. University students. Lancet, 348, 922-925.

[18]. Mc Miller, P., \& Plant, M. (1996). Drinking, smoking and illicit drug use among 15 and 1year old in the United Kingdom. Br Med J, 313, 394-397

[19]. Byrd, R.S., Weitzman, M., \&Doniger, A.S. (1996). Increased drug use among old-for-grade adolescent. Arch PediatrAdolesc, $150,476$.

[20]. Schuckit, MA. (1995). Drug and alcohol use. New York: Plenium medical books company.

[21]. Audu, M.D., Ayuba, L.N., Mela, M., Choji, R.H., \& Sale, S. (2008). Substance abuse and psychiatric morbidity: A 20-Year retrospective study at Jos University Teaching Hospital Nigeria. African Journal of Applied Psychology, 2-4, 95-104

[22]. Namita, P.R., \&Chaturvedi, R.M. (2010). A study of socio-demographic factors contributing to the habit of drug abuse in the urban slum community Mumbai. Biomedical Research, 21(3), 45-50

[23]. Ohaeri, J.., \&Odejide, A.O. (1993). Admissions for drug and alcohol-related problems in Nigerian psychiatric care facilities in one year. Drug and Alcohol Dependence, 3, 101-109

[24]. Adelekan, M.L., \&Adeniran, R.A. (1991). Rehabilitation and Follow-up issues in drug abusers managed at the Neuropsychiatric Hospital, Abeokuta, Nigeria. West African Journal of Medicine, 10(1), $354-360$

[25]. Lawal, R.A., Adelekan, M.L., Ohaeri, J.U., \&Orija O.B. (1998). Rehabilitation of heroin and cocaine abusers managed in a Nigerian psychiatric hospital. East Afr Med J, 75, 108-12

[26]. Dennis-Antwi, J., Adjei, S., Asare, J.B., \&Twene, R.A. (2003). National survey on prevalence and social consequences of substance (drug) use among second cycle and out of school youth in Ghana. Ministry of Health/Ghana Health Services World Health Organization. 
[27]. Hawkins, J.D., Lisher, D.M., Jenson, J.M., \& Catalano, R.F. (1987). Delinquents and drugs: what the evidence suggests about prevention and treatment programming. National Institute on Drug Abuse (DHHS Publication No. ADM 87-1537). Washington, DC: U. S. Government Printing Office.

[28]. Bos, A., Farrell, M., Taylor, C., et al. (2003). Psychiatric morbidity and substance use in young people aged 13-15 years: results from the child and adolescent survey on mental health. British Journal of Psychiatry, 182, 509-517

[29]. Igwe, W.C., \&Ojinn, aka N.C. (2010). Mental health of adolescents who abuse psychoactive substances in Enugu, Nigeria- A cross-sectional study. Italian Journal of Pediatrics, 36, 53

[30]. Laveist, T.A., \& Wallace, J.M. (2000). Health risk and inequitable distribution of liquor stores in African American neighbourhood. Social Science and Medicine, 51, 613-617

[31]. Johnson, J.G., Spitzer, R.L., William, J.B., et al. (1995). Psychiatry comorbidity, health status, and functional impairment associated with alcohol abuse and dependence in primary care patients: Findings of the PRIME MD-1000 study. J Consult. ClinPsychol, 133-140

[32]. Foster, J.H., Peters, T.J., \& Kind, P. (2007). Quality of life, sleep, mood and alcohol consumption: A complex interaction. Addict Biol, 7, 55-65.

[33]. Pettinati, H.M., Gastfriend, D.R., Dong, Q, Kranzler, H.R., et al. (2009). Effects of extended-release naltrexone on quality of life in alcohol-dependent patients. Alcohol Clin Exp Res, 33(2), 350-6

[34]. Srivastava, S., \& Bhatia, M.S. (2009). Quality of life in Alcohol Dependence, depressive, anxiety disorders and healthy controls. Report submitted to Council of Scientific and Industrial Research.

[35]. Connock, M., Juarez-Garcia, A., Jowett, S., et al. (2007). Methadone and buprenorphine for the management of opioid dependence: a systematic review and economic evaluation. Health Technol Assess, 11(9), 1-171

[36]. Astals, M., Domingo-Salvany, A., Buenaventura, C.C., et al. (2008). Impact of substance dependence and dual diagnosis on the quality of life of heroin users seeking treatment. Substance Use Misuse, 43(5), 61-32

[37]. Srivastava, S., Bhatia, M.S., \&Rajender, G., \&Angad, S. (2009). Quality of life in substance use disorders, 12(1), 114-120 